\title{
Psychiatric disorders and general medical conditions: implications for the clinician
}

\author{
P Oosthuizen, P Carey, RA Emsley \\ Department of Psychiatry, University of Stellenbosch, Cape Town, South Africa
}

\begin{abstract}
Patients with severe mental illness have higher than expected prevalence rates of co-morbid general medical conditions, particularly metabolic and cardiovascular disease. They are also at increased risk of contracting HIV. Conversely, these and other medical disorders also increase the risk of developing mental disorders. Mental illness and general medical conditions have mutually adverse effects on long-term outcome. This interaction of diseases contributes significantly to the excess morbidity in and higher than expected standard mortality ratios for patients with mental illness. As medical practice becomes more specialized and arguably compartmentalized it may increasingly fail to integrate health care for patients with severe mental illness. In this paper we discuss the high co-morbidity of mental illness with other medical disorders as well some of the potential mechanisms involved. We furthermore argue that the bidirectional relationship between mental and medical disorders should be considered in the planning of treatment for either group of disorders. The central role of the psychiatrist in co-ordinating and integrating the health care of patients with severe mental illness is emphasized.
\end{abstract}

Key words: Mental illness, Comorbidity, Standard mortality ratio

Received: 15-05-2007

Accepted: 10-12-2007

\section{Introduction}

The rapid expansion of knowledge in the field of medicine is leading to ever-increasing specialization and superspecialization. Medical doctors are finding that there is so much information to assimilate that many are choosing to focus on a particular area and now even on single disease entities. This is also happening in psychiatry, where some specialists now only see and treat patients with particular diseases, such as bipolar disorder. Whereas it is understandable that this is happening, medical practice is running the risk of becoming so compartmentalized that the focus on a particular field of expertise may impair ability to recognize and manage comorbid conditions. It is important for doctors in general to recognize that there is a close relationship and ongoing interaction between mental disorders and general medical conditions. This may be of even greater importance to psychiatrists as they often, for a variety of reasons, become the

\footnotetext{
Correspondence:

DrP Oosthuizen

Dept. Psychiatry, Faculty of Health Sciences, University of Stellenbosch

PO Box 19063, Tygerberg, 7505, South Africa

e-mail: pieto@sun.ac.za
}

primary treating physician to patients with mental illness. In this paper we will use two of arguably the most important comorbidities in the psychiatric setting, namely metabolic syndrome and HIV to discuss the psychiatrist's role in the holistic management of patients with mental illness. Our aim in this paper is to highlight the extent of the relevant problems, and the consequent need for psychiatrists to remain abreast of developments in these disparate clinical fields, in order to provide higher quality care for their patients.

Patients who suffer from mental disorders are at much greater risk of premature death than the general population. ${ }^{1}$ According to the study by Harris et al, the greatest risk is for patients with eating disorders and substance abuse. However, the problem involves many other diagnostic categories as well. For patients with schizophrenia, standard mortality ratios for both natural and unnatural causes are greatly increased. ${ }^{2}$ Although the risk of suicide accounts for some of the risk, the greatest disease burden and cause of mortality in patients with severe mental illness stems from cardiovascular disease. ${ }^{3}$ This holds true not only for psychotic disorders such as schizophrenia, but is also the case in mood disorders. ${ }^{4}$ Furthermore, patients who suffer with depression, bipolar disorder or schizophrenia and who also have a medical disorder have much higher standard mortality ratios than subjects with medical disease alone. ${ }^{5}$ 
Why then is there this striking increase in mortality associated with psychiatric disease and how might this be related to co-morbid medical disease? The answers are inevitably complex, with a number of factors contributing to the increased risk. Some of these factors include:

a) Lifestyle. Patients with severe mental illness are more likely to have sedentary lifestyles, smoke, and make poor dietary choices. ${ }^{6}$ They are less likely to seek help or report symptoms of medical disease.

b) Access to medical care. Because of their disadvantaged position in society, patients with mental illness have poorer access to health care facilities. These patients are less likely to have disposable income to pay for services and/or health insurance and are therefore mostly dependent on the state for medical services. A number of studies have shown that there are significant barriers facing patients with mental illness who want to access medical services and that poor mental health is one of the key predictors of poor access to these services. ${ }^{7,8}$

c) Quality of medical care. Patients who suffer with medical conditions such as diabetes and who also have a psychiatric illness receive poorer care from medical practitioners than other medical patients. These patients are less likely to have regular monitoring of $\mathrm{HbAlc}$ and cholesterol levels and are less likely to have regular eye examinations. They are more likely to have poor long-term glucose and lipid control. ${ }^{9}$ In the Clinical Antipsychotic Trials of Intervention Effectiveness (CATIE) study 30\% of patients with diabetes, $62 \%$ of patients with hypertension and $88 \%$ of patients with dyslipidaemia had received no treatment for their medical condition. ${ }^{10}$ This compares poorly with the treatment for these conditions in the rest of the population, where $46 \%$ of those with dyslipidaemia did not receive treatment. ${ }^{11}$

d) Biological mechanisms. There is growing evidence that there may be shared pathophysiological mechanisms between psychiatric disorders and medical disease. Some of the potential mechanisms involved will be discussed later.

\section{Metabolic and cardiovascular disease}

The global prevalence of chronic diseases is increasing, with the developing world set to carry the largest burden. Although infectious diseases such as malaria and HIV/AIDS remain major health challenges, cardiovascular disease is becoming the major cause of disease burden in the both the developed and the developing world. ${ }^{12}$ Recent findings have shed new light on the enormity of the problem of metabolic disorders and cardiovascular disease in the general population as well as in patients with mental illness, yet at present no universal definition of metabolic syndrome exists. ${ }^{13}$ In fact, multinational societies concerned with obesity, diabetes, cardiovascular disease and the like have in sequential guidelines on the definition and management of metabolic syndrome, placed emphasis on different clinical aspects. ${ }^{14}$ In the most recent of these, the International Diabetes Federation has identified waist circumference as the most important criterion. In their definition, metabolic syndrome is diagnosed through measurement of waist circumference (with norms for men and women representing seven different ethnic groups) plus any two of the following additional criteria: (1) serum triglycerides greater than or equal to $150 \mathrm{mg} / \mathrm{dL}$, or active treatment for hypertriglyceridemia; (2) HDL cholesterol less than $40 \mathrm{mg} / \mathrm{dL}$ for men or less than $50 \mathrm{mg} / \mathrm{dL}$ for women; (3) active antihypertensive treatment or systolic blood pressure greater than or equal to $130 \mathrm{~mm} \mathrm{Hg}$ or diastolic blood pressure greater than or equal to $85 \mathrm{~mm} \mathrm{Hg}$; and (4) fasting glucose greater than or equal to $100 \mathrm{mg} / \mathrm{dL}$, or prior diagnosis of type 2 diabetes mellitus. ${ }^{15}$ In summary, we understand metabolic syndrome to encompass the variable presence of abdominal obesity, hypertension, dyslipidaemia, insulin resistance and diabetes.

In the USA the prevalence of metabolic syndrome is reported to be in the region of $28 \%$, whereas it is only about 9\% in Finland and 14\% in China. ${ }^{16}$ In South Africa the prevalence of obesity and metabolic syndrome is also extremely high. ${ }^{17,18}$ Although we could not find exact prevalence rates for metabolic syndrome, it is known that the prevalence of diabetes in South Africa is as high as 15\% in some population groups. ${ }^{19}$ This would imply an even higher prevalence rate for metabolic syndrome in our patient population.

Although metabolic syndrome and the resultant cardiovascular disease is now being recognized as a major global health problem, the extent of the problem in patients with mental illness has received only scant attention until fairly recently. Studies such as the CATIE trial have highlighted the extent of the problem. In the CATIE trial the prevalence of metabolic syndrome in patients with schizophrenia was almost $43 \%$ at baseline (versus $28 \%$ in the general population). ${ }^{20}$ In another recently published study, the prevalence of metabolic syndrome in patients with severe psychotic and mood disorders was 39\%. ${ }^{21}$ While the CATIE study represents the first large multinational study in which metabolic consequences of use of a wide range of anti-psychotics in "real-life" clinical settings, it is a series of reviews in mood disorders that has begun to generate work on elucidating the possible mechanisms underlying the development of metabolic consequences in psychiatric disease and in response to treatment.

The suggestion that patients with mood disorders are also at risk has been borne out in a number of recent studies and reviews which conclude that mood disorders, including major depressive disorder and bipolar disorder are associated with a two- to threefold increase in the risk for obesity, diabetes and cardiovascular disease. ${ }^{22-24}$ The relationship between depression and cardiovascular disease is a bidirectional one, with depression now recognized as an important cardiovascular risk factor and cardiovascular disease leading to a much increased risk of depressive illness. ${ }^{25,26}$ Depression often predates the first cardiac event, but even more significantly the prevalence of depression in post-myocardial infarction patients is estimated to be around $45 \% .{ }^{27}$ Depression after a myocardial infarction also presents a major risk factor for re-infarction. Furthermore, depression's risk contribution to cardiovascular disease is independent from other, better recognized risk factors such as high cholesterol, hypertension and increased body mass index.

Although this relationship between depression and cardiovascular disease has been known for some time, the exact mechanisms are not quite clear. Whereas the issues of poor lifestyle, access to care and quality of medical care are 
important, there is growing evidence that there may also be biological mechanisms underlying this interaction. These mechanisms include hypothalamic-pituitary-adrenal axis dysregulation, reduced heart rate variability, altered blood platelet functioning, reduced baroreflex sensitivity and impaired immune functioning. Another recently proposed shared mechanism involves the role of inflammatory processes. It seems increasingly likely that some psychiatric disorders such as Alzheimer's disease, depression and schizophrenia may have inflammatory processes as part of their pathophysiology, much like cardiovascular disease. ${ }^{28-30}$ Pro-inflammatory amino-acids, such as homocysteine may therefore present a shared risk factor or a marker of a shared risk factor (chronic inflammation) for a number of psychiatric disorders and associated cardiovascular/metabolic disorders.

\section{HIV}

The close relationship between HIV/AIDS and mental illness is of particular importance to South African psychiatrists. Health services in the developed world have long realized the link between HIV/AIDS and mental illness and have integrated the approach to the two conditions. In the developing world this has not yet happened on a satisfactory scale. HIV confers a considerable physical and psychological burden on those it infects. Depression, the most frequently encountered manifestation of this psychological burden is present in 34.9\% in HIV clinic attendees ${ }^{31}$ and up to 50\% of community HIVpositive people. ${ }^{32}$ Sub-Saharan Africa is currently the worst affected region with upwards of $60 \%$ of worlwide infections (34.5 million), while Southern Africa is the worst affected within this region. ${ }^{33}$ From this it follows that a considerable burden of depression exists within this region that at present is largely untreated. This assertion follows data suggesting that treatment of depression in routine primary care clinics is poor $^{34}$ and is likely to be worse in clinics focused on chronic medical illnesses including HIV. ${ }^{35}$ There are a number of difficulties with the accurate diagnosis of depression in HIV including both overlap with HIV symptoms and certain sideeffects of HIV medications. Aside from the burden of untreated depression in general, a number of other compelling reasons exist as to why depression should be accurately identified and aggressively treated in people with HIV. First, poor identification of depression appears to have direct consequences for accelerated HIV disease progression. ${ }^{36}$ Furthermore, evidence suggests that depression is independently associated with increased HIV mortality and is associated with a more rapid decline in CD4+ lymphocyte counts. ${ }^{37}$ The sheer extent of the HIV pandemic is placing a considerable burden on health related spending in South Africa with the result that highly active antiretroviral therapy (HAART) is only available to those with CD4 counts $<200 \mathrm{cell} s / \mathrm{mm}^{3}$ or alternatively WHO stage IV disease. ${ }^{38}$ Combining evidence of the high prevalence of depression in people recently diagnosed with HIV $^{39}$ with the effects on progression of HIV disease, it would seem that early and effective intervention for depression may reduce healthcare costs by improving quality of life and delaying disease progression and the need for costly HAART. When HAART treatment is inevitably introduced, improved treatment adherence is likely with more effectively treated depression. ${ }^{40,41}$
Second, depression and stress increase central nervous system (CNS) neuropeptides including substance P. ${ }^{42}$ Substance P has been implicated in the pathophysiology of numerous neuropsychiatric disorders including depression. ${ }^{43}$ Evidence now suggests that receptors for substance P (neurokinin-1) are most widely expressed in brain regions involved in affective regulation. ${ }^{44}$ As such it is understandable how affective dysregulation, probably mediated by subtance P and other neuropeptides, can have a direct effect on immunomodulaton and hence on HIV disease progression. ${ }^{45}$ This is supported by findings that subtance P stimulates cytokine production including TNF- and upregulates HIV expression on T cell $\mathrm{s}^{46}$ and other crucial targets for the HI virus. ${ }^{47}$ While SSRI treatment of depression does not specifically target neuropeptide receptors, recent preliminary evidence suggests that higher pre-treatment serum substance P levels are associated with clinical response to SSRIs in depression. ${ }^{48}$ It remains to be investigated whether SSRI treatment in asymptomatic HIV delays disease progression in association with levels of substance P. To what extent substance $\mathrm{P}$ is a reliable predictor of treatment response to SSRIs in HIV requires further exploration. Once again, the relationship between HIV and psychiatric illness is a bidirectional one. Just as patients with HIV are at increased risk of depression and other psychiatric disorders, severe mental illness increases the risk of contracting HIV.49 Patients with severe mental illness are less likely to use condoms and more likely to have multiple sexual partners. Due to their isolated position in society, they are more likely to be victims of sexual coercion. Cognitive deficits as a result of mental illness may also exacerbate impulsive behaviours and poor judgment. ${ }^{50}$

\section{The role of psychotropic medication}

There is some evidence to suggest that patients with severe mental illness are at greater risk of developing abnormal glucose homeostasis independent of treatment with medication. ${ }^{51}$ The recent literature has however mostly focused on the effects of psychotropic medications on metabolic and cardiovascular health. Some evidence is emerging for the potential effects of antidepressants on the parameters of weight and metabolic health ${ }^{52-54}$, although it is somewhat surprising to see the relative lack of information and studies with antidepressants when compared to the anti-psychotics. From the information currently available, it seems that there is a highly variable effect, with certain SSRI's such as paroxetine seemingly more likely to have adverse metabolic effects than other SSRI's. ${ }^{54}$ Mirtazapine, a so-called NaSSA antidepressant, causes weight gain, but apparently does not necessarily affect glucose homeostasis. ${ }^{53}$ In contrast a great number of recent studies have focused on the metabolic and cardiovascular effects of anti-psychotic medications. Once again, there is relatively little information on the effects of the first generation anti-psychotics, but there is some evidence that chlorpromazine may cause metabolic problems similar to those caused by some of the second generation antipsychotics. ${ }^{55}$ Although there are many studies with different outcomes that are hotly debated in the literature, a pattern seems to be emerging: clozapine and olanzapine seem to have to the most marked effects on metabolic parameters, followed by risperidone and quetiapine. Ziprasidone and aripiprazole do not thus far seem to have 
adverse effects on metabolic health. ${ }^{56}$ Guidelines for metabolic monitoring of patients on anti-psychotics are emerging, but as virtually all of the studies and recommendations are from the developed world, it is not clear to what extent these guidelines are transferable to the developing world. There are large variations in the prevalence of metabolic syndrome in different population groups, and recent studies have again shown that there are also large differences between groups of patients with schizophrenia from different countries in the developed world. ${ }^{57}$

\section{Implications for the clinician}

Psychiatrists understandably prefer to focus on the diagnosis and management of mental disorders. However these conditions are so intimately interwoven with other, non-mental disorders that it has become imperative for psychiatrists to be more aware of these co-morbid conditions. Furthermore, psychiatrists should keep these conditions in mind when they assess their patients and particularly when they decide on treatment, as the treatments chosen for psychiatric disorders may also affect other conditions. This is of particular concern when prescribing anti-psychotic medication, but may also be relevant in the prescribing of antidepressants and mood stabilizers. But it implies more than just considering the potential side-effects of medication. Patients with mental illness are poorly served by the general medical services. These patients have the right to a co-ordinating, integrating health provider. Normally, this responsibility would fall to the general practitioner, but the literature shows that this is not happening for patients with severe mental illness. By default then, it seems increasingly that the psychiatrist is the person most likely - and arguably the most suitable - to fulfill this role.

In the South African health system, psychiatry has, to a significant degree, been compartmentalized and separated from the rest of medicine. The majority of psychiatric inpatients are admitted to large psychiatric hospitals where severe limitations are placed on the budgets for special investigations. Registrars are actively discouraged from doing "unnecessary" special investigations and routine monitoring of metabolic parameters are not currently considered as essential. Psychiatric hospitals stock very limited supplies of medications for the treatment of general medical conditions. All general medical issues are supposed to be dealt with by the general hospitals. Given that access to the general hospitals for inpatients from a psychiatric hospital is only available for emergencies and those with acute, life-threatening medical illnesses, issues such as the monitoring of metabolic health are not seen as a priority. The unfortunate result of this is that the need for the general medical care of the psychiatric patient goes unnoticed and remains unattended. Once discharged, patients with psychiatric disorders are expected to collect their medication from overcrowded community clinics, where they are only seen briefly and often only to issue their next month's supply of medication. Little or no monitoring of medical conditions for these patients occurs at this level.

Although we advocate a more integrated approach, this does not mean that psychiatrists have to take over the management of medical disorders, but rather that the psychiatrist should co-ordinate the mental and physical health care of his/her patient by asking patients about these, liaising with colleagues from other medical specialties, promoting healthy lifestyles, conducting psycho-education in relation to medical disorders and promoting medication compliance and attendance of follow-up appointments. Psychiatrists can also play an important role in monitoring metabolic health and advocating access to better medical care for our patients, including access to antiretroviral therapy. Numerous questions specific to our environment arise in patients co-morbid for psychiatric and medical conditions, and specific research is needed to address these. For instance, very little is known about the co-occurrence of medical and psychiatric disorders in the developing world. Before we advocate first-world style monitoring of metabolic health in our psychiatric population, we need to ascertain whether the problem actually exists in these settings and if so, to what extent. Similarly, differential impact of HIV infection and susceptibility to severe mental illness and dementia remain largely unanswered at this time.

\section{References}

1. Harris EC, Barraclough B. Excess mortality of mental disorder. Br J Psychiatry 1998;173: 11-53.

2. Brown $S$, Barraclough B, Inskip H. Causes of the excess mortality of schizophrenia. Br J Psychiatry 2000;177: 212-217.

3. Osborn DPJ, Levy G, Nazareth I, et al. Relative Risk of Cardiovascular and Cancer Mortality in People With Severe Mental Illness From the United Kingdom's General Practice Research Database. Arch Gen Psychiatry 2007;64: 242-249.

4. Joukamaa M, Heliovaara $M$, Knekt $P$, et al. Mental disorders and cause-specific mortality. Br J Psychiatry 2001;179: 498-502.

5. Newcomer JW. Medical risk in patients with bipolar disorder and schizophrenia. Journal of Clinical Psychiatry 2006;67: 25-30.

6. Brown $S$, Birtwistle J, Roe L, et al. The unhealthy lifestyle of people with schizophrenia. Psychological Medicine 1999;29: 697-701.

7. Bauer MS, Williford WO, McBride L, et al. Perceived barriers to health care access in a treated population. International Journal of Psychiatry in Medicine 2005;35: 13-26.

8. Studts CR, Stone RF, Barber GM. Predictors of access to health-care services among groups of TANF recipients in Kentucky. Social Service Review 2006;80: 527-548.

9. Frayne SM, Halanych JH, Miller DR, et al. Disparities in diabetes care - Impact of mental illness. Archives of Internal Medicine 2005; 165: 2631-2638.

10. Nasrallah HA, Meyer JM, Goff DC, et al. Low rates of treatment for hypertension, dyslipidemia and diabetes in schizophrenia: Data from the CATIE schizophrenia trial sample at baseline. Schizophrenia Research 2006;86: 15-22.

11. Goff DC, Jr., Bertoni AG, Kramer H, et al. Dyslipidemia Prevalence, Treatment, and Control in the Multi-Ethnic Study of Atherosclerosis (MESA): Gender, Ethnicity, and Coronary Artery Calcium. Circulation 2006;113: 647-656.

12. Kengne AP, Amoah AGB, Mbanya JC. Cardiovascular complications of diabetes mellitus in sub-Saharan Africa. Circulation 2005;112: 3592-3601.

13. Pi-Sunyer X. The Metabolic Syndrome: How to Approach Differing Definitions. Medical Clinics of North America 2007;91: 1025-1040.

14. Brietzke SA. Controversy in Diagnosis and Management of the Metabolic Syndrome. Medical Clinics of North America 2007;91: 1041-1061.

15. Alberti KGMM, Zimmet P, Shaw J. Metabolic syndrome-a new worldwide definition. A Consensus Statement from the International Diabetes Federation. Diabetic Medicine 2006;23: 469-480.

16. Reynolds K, He J. Epidemiology of the Metabolic Syndrome. 
American Journal of the Medical Sciences 2005:330: 273-279.

17. Ntyintyane LM, Panz VR, Raal FJ, et al. Metabolic syndrome, undiagnosed diabetes mellitus and insulin resistance are highly prevalent in urbanised South African blacks with coronary artery disease. Cardiovasc J S Afr 2006; 17: 50-55.

18. van der Merwe MT, Pepper MS. Obesity in South Africa. Obesity Reviews 2006; 7: 315-322.

19. Society for endocrinology, metabolism and diabetes of South Africa. Prevalence of type 2 diabetes in different South African population groups. 2007.

20. McEvoy JP, Meyer JM, Goff DC, et al. Prevalence of the metabolic syndrome in patients with schizophrenia: Baseline results from the Clinical Antipsychotic Trials of Intervention Effectiveness (CATIE) schizophrenia trial and comparison with national estimates from NHANES III. Schizophrenia Research 2005;80: 19-32.

21. Bermudes RA, Keck PE, Welge JA. The prevalence of the metabolic syndrome in psychiatric inpatients with primary psychotic and mood disorders. Psychosomatics 2006;47: 491-497.

22. Taylor V, MacQueen G. Associations between bipolar disorder and metabolic syndrome: A review. Journal of Clinical Psychiatry 2006;67: 1034-1041.

23. Kahl KG, Schweiger U. The metabolic syndrome and diabetic risk in psychiatric patients. Epidemiology, risk factors, and monitoring Psychopharmakotherapie 2006;13: 43-+.

24. McElroy SL, Kotwal R, Malhotra S, et al. Are mood disorders and obesity related? A review for the mental health professional. Journal of Clinical Psychiatry 2004;65: 634-651.

25. Grippo AJ, Johnson AK. Biological mechanisms in the relationship between depression and heart disease. Neuroscience and Biobehavioral Reviews 2002;26: 941-962.

26. Grippo AJ, Beltz TG, Johnson AK. Behavioral and cardiovascular changes in the chronic mild stress model of depression. Physiology \& Behavior 2003;78: 703-710.

27. Schleifer SJ, Macarihinson MM, Coyle DA, et al. The Nature and Course of Depression Following Myocardial-Infarction. Archives of Internal Medicine 1989;149: 1785-1789.

28. Kemperman RFJ, Veurink $M$, van der Wal T, et al. Low essential fatty acid and B-vitamin status in a subgroup of patients with schizophrenia and its response to dietary supplementation. Prostaglandins Leukotrienes and Essential Fatty Acids 2006;74: 75-85.

29. Lerner V, Miodownik C, Kaptsan A, et al. High serum homocysteine levels in young male schizophrenic and schizoaffective patients with tardive parkinsonism and/or tardive dyskinesia. Journal of Clinical Psychiatry 2005;66: 1558-1563.

30. Justo D. Smoldering systemic inflammation: a link between schizophrenia, smoking and obesity. Acta Psychiatrica Scandinavica 2006;1 13: 245-246.

31. Olley BO, Seedat S, Stein DJ. Psychopathology and coping in recently diagnosed HIVIAIDS patients. S Afr Med J 2004;94: 720, 722

32. Bing EG, Burnam MA, Longshore D, et al. Psychiatric disorders and drug use among human immunodeficiency virus-infected adults in the United States. Arch Gen Psychiatry 2001;58: 721-728.

33. UNAIDSI WHO AIDS epidemic update. WHO . 2004.

34. Carey PD, Stein DJ, Zungu-Dirwayi N, et al. Trauma and posttraumatic stress disorder in an urban Xhosa primary care population: prevalence, comorbidity, and service use patterns. $J$ Nerv Ment Dis 2003;1 191: 230-236.

35. Evans DL, Staab J, Ward H, et al. Depression in the medically ill: management considerations. Depress Anxiety 1996;4: 199-208.

36. Leserman J, Jackson ED, Petitto JM, et al. Progression to AIDS: the effects of stress, depressive symptoms, and social support.
Psychosom Med 1999;61: 397-406.

37. Ickovics JR, Hamburger ME, Vlahov D, et al. Mortality, CD4 cell count decline, and depressive symptoms among HIV-seropositive women: longitudinal analysis from the HIV Epidemiology Research Study. JAMA 2001;285: 1466-1474

38. Department of Health. National Antiretroviral Treatment Guidelines. 2004. Jacana.

39. Olley BO, Seedat S, Stein DJ. Psychopathology and coping in recently diagnosed HIVIAIDS patients. S Afr Med J 2004;94: 720, 722

40. Ammassari A, Antinori A, Aloisi MS, et al. Depressive symptoms, neurocognitive impairment, and adherence to highly active antiretroviral therapy among HIV-infected persons. Psychosomatics 2004;45: 394-402.

41. Gordillo V, del AJ, Soriano V, et al. Sociodemographic and psychological variables influencing adherence to antiretroviral therapy. AIDS 1999; 13: 1763-1769.

42. Fehder WP, Sachs J, Uvaydova M, et al. Substance P as an immune modulator of anxiety. Neuroimmunomodulation 1997;4: 42-48.

43. Stout SC, Owens MJ, Nemeroff CB. Neurokinin(1) receptor antagonists as potential antidepressants. Annu Rev Pharmaco. Toxicol 2001;41:877-906.: 877-906.

44. Harrison S, Geppetti P. Substance p. Int J Biochem Cell Biol 2001;33: 555-576.

45. Evans DL, Ten Have TR, Douglas SD, et al. Association of depression with viral load, CD8 T lymphocytes, and natural killer cells in women with HIV infection. Am J Psychiatry 2002; 159: 1752-1759

46. Folks TM, Justement J, Kinter A, et al. Cytokine-induced expression of HIV-1 in a chronically infected promonocyte cell line. Science 1987;238: 800-802

47. Li Y, Douglas SD, Song L, et al. Substance P enhances HIV-1 replication in latently infected human immune cells. J Neuroimmunol 2001;121: 67-75.

48. Lieb K, Walden J, Grunze H, et al. Serum levels of substance $P$ and response to antidepressant pharmacotherapy. Pharmacopsychiatry 2004;37: 238-239

49. Meade CS, Sikkema KJ. HIV risk behavior among adults with severe mental illness: A systematic review. Clinical Psychology Review 2005;25: 433-457

50. Collins PY, Holman AR, Freeman MC, et al. What is the relevance of mental health to HIVIAIDS care and treatment programs in developing countries? A systematic review. Aids 2006;20: 1571-1582.

51. Haupt DW, Newcomer JW. Abnormalities in glucose regulation associated with mental illness and treatment. Journal of Psychosomatic Research 2002;53: 925-933.

52. Chadwick W, Wilson $G$, van de Venter $M$, et al. Shifts in metabolic parameters surrounding glucose homoeostasis resulting from tricyclic antidepressant therapy: implications of insulin resistance? Journal of Pharmacy and Pharmacology 2007;59: 95-103.

53. Laimer M, Kramer-Reinstadler K, Rauchenzauner M, et al. Effect of mirtazapine treatment on body composition and metabolism Journal of Clinical Psychiatry 2006;67: 421-424.

54. Raeder MB, Bjelland I, Vollset SE, et al. Obesity, dyslipidemia, and diabetes with selective serotonin reuptake inhibitors: The Hordaland Health Study. Journal of Clinical Psychiatry 2006;67: 1974-1982.

55. Haupt DW, Newcomer JW. Hyperglycemia and antipsychotic medications. Journal of Clinical Psychiatry 2001;62: 15-26.

56. Newcomer JW. Metabolic considerations in the use of antipsychotic medications: A review of recent evidence. Journal of Clinical Psychiatry 2007;68: 20-27.

57. Bobes J, Arango C, Aranda P, et al. Cardiovascular and metabolic risk in outpatients with schizophrenia treated with antipsychotics: Results of the CLAMORS Study. Schizophrenia Research 2007;90: 162-173. 